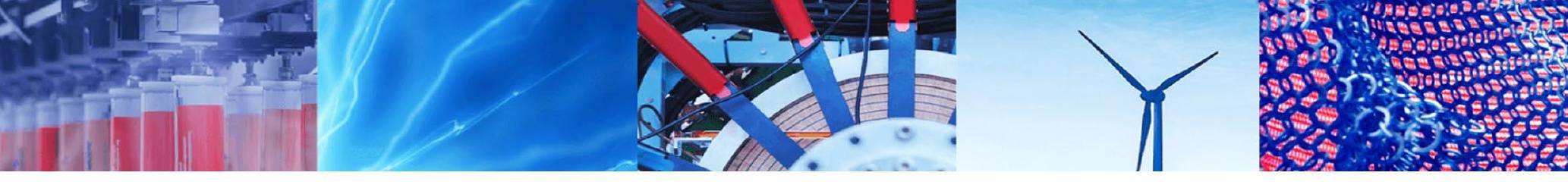

Research Article

\title{
Optimization of strain measurement procedure based on fuzzy quality evaluation and Taguchi experimental design
}

\author{
Mehdi Moayyedian $^{1} \cdot$ Javad Farrokhi Derakhshandeh ${ }^{1} \cdot$ Sang Heon Lee ${ }^{2}$
}

C) Springer Nature Switzerland AG 2019

\begin{abstract}
The reliability of the measurement system, accuracy, and precision are significant criteria at any measurement procedure. Strain gage sensors are one of the tools to measure the strain of structures in engineering applications. Different controllable and extraneous parameters will change the accuracy and precision of the strain gauges. As a result, it is valuable to investigate the most significant parameters and the percentage of their contributions affecting the strain measurement. This paper presents the multi-objective optimization in strain gauge measurements to determine the Reliability and Noise indices. The best set of parameters with a high-reliability index (which means low noise index) for strain measurement based on two significant objectives of accuracy and precision are evaluated. The significant parameters, their optimal levels and percentage of contributions are identified according to the Taguchi and Fuzzy Analytic Hierarchy Process (FAHP) with Technique for the Order Performance by its Similarity to the Ideal Solution (TOPSIS) methods. To obtain this evaluation, three levels $\left(L_{1}, L_{2}\right.$, and $\left.L_{3}\right)$ of temperature $\left(P_{1}\right)$, the length of wires $\left(P_{2}\right)$, and the point of applied force $\left(P_{3}\right)$ are considered. This configuration leads to $L_{9}$ orthogonal array of Taguchi approach based on the number of parameters and their levels. The triangular membership function for the weightiness of accuracy and precision is used via AHP. By using the Taguchi method, the optimum set of parameters are identified as $P_{1}$ at $L_{1}, P_{2}$ at $L_{2}$, and $P_{3}$ at $L_{3}$ to have a higher reliability index compared to trial 8 . By applying the optimum design of experiment, it is found that the reliability index is $95 \%$. The results also show that the highest reliability index is related to trial 8 with $87.65 \%$ reliability. Finally, the combination of FAHP and TOPSIS with Taguchi method for multi-objective optimization is introduced and proposed as a practical and useful model to determine the best set of parameters with the highest reliability index in strain measurement.
\end{abstract}

Keywords Strain measurement · Accuracy · Precision · TOPSIS · Fuzzy · FAHP · Taguchi · Reliability index

\section{Introduction}

Piezo-resistivity or change in the electrical resistivity can be considered as one of the widely used physical phenomena at different kind of sensory applications. Strain gauges are useful and valuable devices to measure the time-dependent magnitude of deformation and can be associated with determining the stresses of the structure in a wide range of applications $[5,11-13,19,21,23,34]$. To achieve this, a deep knowledge of mechanical engineering is required for modeling calculation to a constitutive equation for the stress-strain relationship [22, 33]. The basic function of the strain gauge is based on transforming the strain in a certain direction to change its electric resistance $[20,38]$. While the strain gauges have been employed for a few decades, still there is little guidance on how to evaluate mechanical properties and the aspects of strain measurement have been covered in brief [22, 31, 33].

There are several uncertainties in measuring the strain of the object, which can be related to the geometry,

$\triangle$ Mehdi Moayyedian, mehdi.moayyedian@aum.edu.kw $\mid{ }^{1}$ College of Engineering and Technology, American University of the Middle East, Kuwait City, Kuwait. ${ }^{2}$ School of Engineering, University of South Australia, Mawson Lakes Campus, Mawson Lakes, SA 5095, Australia.

SN Applied Sciences (2019) 1:1392 | https://doi.org/10.1007/s42452-019-1428-x

Received: 25 June 2019 / Accepted: 9 October 2019 / Published online: 14 October 2019 
material, and optical of the test case. Besides, other uncertainties which can be considered in analyzing the system are related to the methods of measurement such as configuration, the number, and distribution of measuring points, the number of sampling, and the operator $[8,40]$. Davis [8] conducted an excellent study using different techniques of strain measurement during tensile testing. It was found that the size of the sample, environmental conditions, measurement requirement for accuracy and precision of anticipated strain levels are important factors and can significantly affect the deformation of the structure [8]. Therefore, it was found that a precise optimization method is required to characterize the tensile testing of materials properly.

To have a product with high quality and low-cost, manufacturing companies typically attempt to produce valuable products in a short time to compete with others. For a complex product, the design of the product requires optimization tasks comprising multiple objectives. This optimization can be considered both in numerical simulations and experimental measurements. In computational simulations, for instance, it will take more than $100 \mathrm{~h}$ to run one crash simulation for a non-very complicated model $[16,39]$. For a two-variable optimization problem, assuming on average 50 iterations are needed by optimization and assuming each iteration needs one crash simulation, the total computation time would be more than 2 months. This type of study is mostly associated with trial and error. Trial and error has been identified as the main methodology in problem solving and has been employed extensively, in particular, in numerical modeling $[1,4,9,10,16]$. Therefore, to decrease the number of test case studies, the design of experiments and optimization of measurement are valuable skills, which can be employed to enhance productivity and efficiency.

One of the optimization method, which is implemented for quality evaluation in industry is the Design of Experiment (DOE) and using relevant tools such as Taguchi method, Signal to noise $(S / N)$ ratio and Analysis of Variance (ANOVA) $[2,25,28]$. One of the most advantage of DOE is to find the significant parameters and their optimum level. This assists to find the best quality evaluation of the products. Amer et al. [3] showed that DOE can be considered as a valuable tool in order to define the right parameters and levels in injection moulding with less internal and external defects. The effect of cutting fluid in manufacturing process also can be optimized by DOE. Sarikaya and Güllü [35] found to have a lower rate of tool wear pattern and surface roughness, the most significant parameters and their levels are vegetable based cutting fluid with $180 \mathrm{~mL} / \mathrm{h}$ fluid flow rate and $30 \mathrm{~m} / \mathrm{min}$ cutting speed. Besides, the surface roughness was investigated for turning machine and the results indicated that the most significant parameter was feed rate on the surface roughness [35]. Later, Sarikaya et al. [36] showed that DOE is able to reduce the amount of oil used for machining purposes with an improvement in machinability of material. Also, in drilling process, the most significant parameters affecting the dimensional accuracy is cutting speed for both coated and uncoated tools using DOE [24].

Two conventional methods which determine the practical and possible factors in increasing the quality in different applications are Fuzzy logic and TOPSIS. It was shown that the combination of these methods can be used in injection molding to find the optimum set of parameters and reduce the number of internal and external defects $[17,29]$. Consequently, choosing the wrong set of geometrical and process parameters will decrease the mechanical properties of the injected part $[26,27]$. The Taguchi method and DOE can be employed and used as tools for single and not for multi-objective problems. To guarantee that different internal and external defects can be controlled appropriately, the fuzzy logic theory can be proposed [6]. It was shown that in injection molding, in order to find the best set of process parameters, the combination of fuzzy and TOPSIS can be employed, which has the high level of moldability for producing the product $[7,29,30,32]$.

There is an extensive investigations which implemented the TOPSIS concept for optimization process. For instance, in micromachining procedure, to have an accurate result, TOPSIS was applied [15]. To determine the effect of different input parameters on the machining accuracies, two types of fluids were employed. Hence, sodium chloride was selected as a fluid with higher material removal rate using TOPSIS [15]. Another application of TOPSIS method is related to the surface performance measurements in electrical discharge machining process. Huo et al. [18] found that magnitude of the current has the most influence on the performance measurement of the process.

The above review intimates that that although the integration of FAHP with TOPSIS and AHP with FTOPSIS are useful methods for quality evaluation at different engineering applications, they are unable to cover all different possibilities to determine the optimum level and the contribution rate of significant controllable factors. To the best of the author's knowledge, there is no published study employing Taguchi with FAHP and TOPSIS in strain measurement to evaluate the optimum parameters of DOE. Consequently, the objective of this paper is to evaluate and determine the most significant parameters affecting the reliability of strain gauges for different industrial applications. This paper investigates and proposed a new approach for the multi-objective optimization method in strain measurement based on two significant objectives comprising accuracy and precision. Thus, a new approach 
based on Taguchi with FAHP and TOPSIS for quality evaluation of strain measurement process is developed and introduced. There are controllable and extraneous parameters to evaluate the final quality of any process. One of the main application of Taguchi method is to enhance the control of the parameters. In this paper, three controllable parameters namely the temperature, the length of wire and the point of application are considered. Besides, uncontrollable parameters such as operator which leads to produce noises is noted.

In general, the Taguchi method is applied for a single objective. So, the novelty of the paper is to determine the reliability index in strain measurement based on two objectives (accuracy and precision) via Fuzzy Analytic Hierarchy Process (FAHP) and TOPSIS. Finally, by having a single objective (Reliability index), Taguchi method is implemented to find the most significant parameters, their optimum levels, and their contribution to find the highest reliability index.

\section{Proposed methodology to enhance the quality of measurement}

A comprehensive methodology is developed and introduced herein by applying FAHP along with TOPSIS and Taguchi that aims to optimize the multi-objective process in strain measurement. The proposed approach determines the best alternatives with higher reliability, as shown in Fig. 1.
Fig. 1 Flowchart of optimization of strain measurement based on fuzzy approach and the Taguchi method

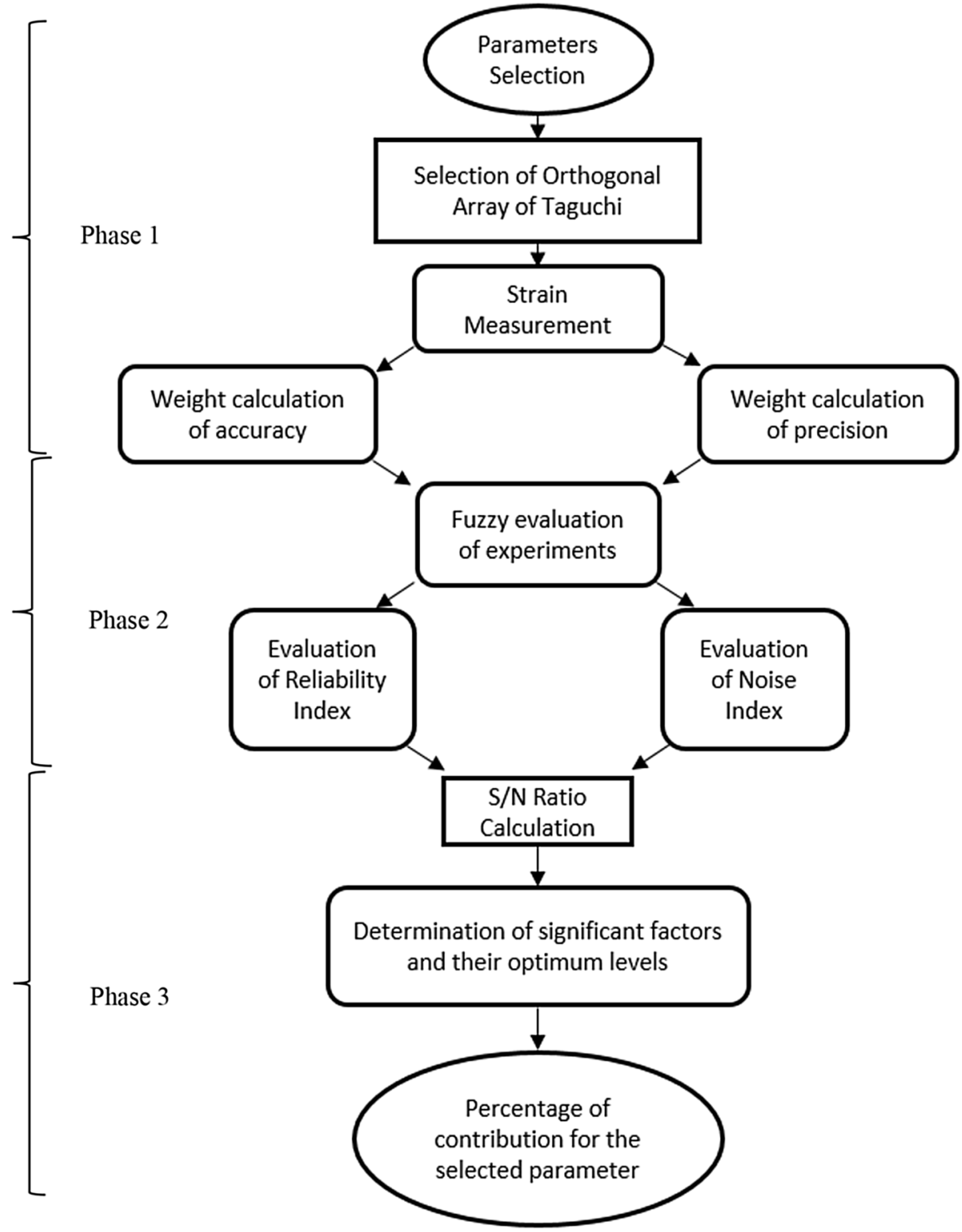


The objective of this paper is to determine the significant parameters affecting the reliability of strain gauges.

\subsection{Problem description}

Two common objectives in strain measurement which evaluate the reliability of the measurement procedure are defined as precision and accuracy. These two objectives are significantly related to different controllable parameters. The accuracy of the measurement is the difference between the measured value and the real value. Any deviation of the measured value from the real value is the error within the system $[31,37]$. Furthermore, the precision is the closeness of two or more measurement to each other. By employing these two criteria, the reliability of strain measurement is determined.

For rating the seriousness of each criterion, a fuzzy evaluation method is applied. Five different levels are defined, namely $\tilde{\alpha}_{1}, \tilde{\alpha}_{2}, \tilde{\alpha}_{3}, \tilde{\alpha}_{4}$, and $\tilde{\alpha}_{5}$. The seriousness of each criterion is evaluated by applying linguistic terms named as very low, low, medium, high, and very high. For rating the seriousness of precision and accuracy, the triangle membership functions with triplet descriptions are used as shown in Fig. 2 and Table 1, respectively [6, 30].

\subsection{Weight calculation of state variables}

For weighting the selected parameters, Analytic Hierarchy Process (AHP) is implemented based on the weight profit factor [7].

The vector $S\left(x_{j}\right)=\left\{s_{1}\left(x_{j}\right), s_{2}\left(x_{j}\right), \ldots, s_{p}\left(x_{j}\right)\right\}$ is given as follow:

$s_{j}\left(x_{j}\right)= \begin{cases}\lambda_{1} \alpha & x_{j}=\tilde{\alpha}_{1} \\ \alpha & x_{j}=\tilde{\alpha}_{2} \\ 1 & x_{j}=\tilde{\alpha}_{3} \\ \beta & x_{j}=\tilde{\alpha}_{4} \\ \lambda_{2} \alpha & x_{j}=\tilde{\alpha}_{5}\end{cases}$

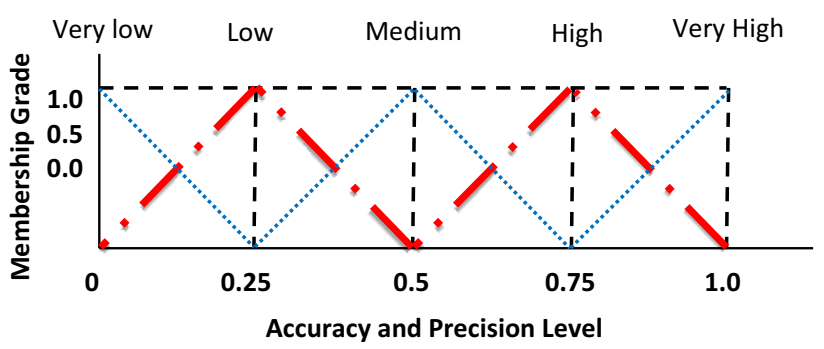

Fig. 2 Triangular membership function for the weightiness of accuracy and precision
Table 1 Triplet description of Linguistic variables for the evaluation of accuracy and precision weightiness associated with Fig. 1

\begin{tabular}{lll}
\hline Linguistic variables & Fuzzy rating & Triple description \\
\hline Very high & $\mathrm{a}_{5}$ & $(0.75,1,1)$ \\
High & $\mathrm{a}_{4}$ & $(0.5,0.75,1)$ \\
Medium & $\mathrm{a}_{3}$ & $(0.25,0.5,0.75)$ \\
Low & $\mathrm{a}_{2}$ & $(0,0.25,0.5)$ \\
Very low & $\mathrm{a}_{1}$ & $(0,0,0.25)$ \\
\hline
\end{tabular}

where $\alpha, \beta$, and $\lambda$ are positive, negative, and regulative factors, respectively.

\subsection{Variable weight definition and implementation}

The initial weights for accuracy and precision are implemented via AHP. The variable weight vector $W$ is the normalized product of constant weight factor $w$ and variable weight state vector $s$ which can be evaluated based on Eq. 2.

$W_{j}\left(x_{j}\right)=\frac{w_{j} s_{j}\left(x_{j}\right)}{\sum_{k=1}^{n} w_{k} s_{k}\left(x_{j}\right)}$

\subsection{Taguchi orthogonal array}

Taguchi is a technique to reduce the number of experiments which create robustness for different industrial applications. Hence, there is a huge reduction of time and cost throughout the manufacturing process [14]. In this paper, the interaction of Taguchi with TOPSIS and FAHP is implemented to investigate the highest reliability index throughout the strain measurement procedure. Selected parameters and their relevant levels are listed and introduced, as shown in Table 2.

Based on the number of parameters and their levels, the $L_{9}$ orthogonal array is created as shown in Table 3.

\subsection{TOPSIS}

In this study, $m$ represent the trial number of experiments number $n$ represent different objectives for quality

Table 2 Three levels of the temperature $\left(P_{1}\right)$, the length of wires $\left(P_{2}\right)$, and the point of applications $\left(P_{3}\right)$

\begin{tabular}{llcl}
\hline & $P_{1}\left({ }^{\circ} \mathrm{C}\right)$ & $P_{2}(\mathrm{~cm})$ & $P_{3}(\mathrm{~cm})$ \\
\hline Level 1 & 23 & 5 & 1 \\
Level 2 & 37 & 10 & 2 \\
Level 3 & 50 & 15 & 3 \\
\hline
\end{tabular}


Table $3 L_{9}$ orthogonal array of Taguchi

\begin{tabular}{llll}
\hline Trial number & $P_{1}$ & $P_{2}$ & $P_{3}$ \\
\hline 1 & 1 & 1 & 1 \\
2 & 1 & 2 & 2 \\
3 & 1 & 3 & 3 \\
4 & 2 & 1 & 2 \\
5 & 2 & 2 & 3 \\
6 & 2 & 3 & 1 \\
7 & 3 & 1 & 3 \\
8 & 3 & 2 & 1 \\
9 & 3 & 3 & 2 \\
\hline
\end{tabular}

evaluation. Initial weight calculation is implemented via $\mathrm{AH}$. Then, fuzzy relative matrix based on the seriousness of accuracy and precision is applied and different weight of each criterion is considered by Eqs. 1 and 2. Finally, the varied weighted fuzzy evaluation matrix is based on Eq. 3 as follow:

$\tilde{V}=\left[\tilde{v}_{i j}\right]_{m \times n} \quad i=1,2, \ldots, m$ and $j=1,2, \ldots, n$

here $\tilde{v}_{i j}=\tilde{r}_{i j} \times W_{j}=\left(r_{i j 1} W_{j}, r_{i j 2} W_{j}, r_{i j 3} W_{j}\right)$.

TOPSIS is applied to rank the order of 9 experiments. Based on the selected fuzzy decision matrix, it is evident that the elements $\tilde{v}_{i j}$ of normalized positive triangle numbers and their range is related to interval $[0,1]$. Hence, the definition of fuzzy positive ideal solution (FPIS) and fuzzy negative ideal solution (FNIS) is defined as:

$A^{+}=\left\{\tilde{v}_{1}^{+} \tilde{v}_{2}^{+} \ldots \tilde{v}_{n}^{+}\right\}$

$A^{-}=\left\{\tilde{v}_{1}^{-} \tilde{v}_{2}^{-} \ldots \tilde{v}_{n}^{-}\right\}$.

where $\tilde{v}_{j}^{+}=\left(v_{j}^{+}, v_{j}^{+}, v_{j}^{+}\right), \tilde{v}_{j}^{+}=\max \left(v_{i j}^{+}\right), \tilde{v}_{j}^{-}=\left(v_{j}^{-}, v_{j}^{-}, v_{j}^{-}\right)$, and $v_{j}^{-}=\min \left(v_{i j}^{-}\right)$.

The distance of each alternative or experiment based on the orthogonal array can be calculated by following equations:

$d_{i}^{+}=\sum_{j=1}^{n} d\left(\tilde{v}_{i j}, \tilde{v}_{j}^{+}\right), \quad \forall i=1,2, \ldots, m$

$d_{i}^{-}=\sum_{j=1}^{n} d\left(\tilde{v}_{i j}, \tilde{v}_{j}^{-}\right), \quad \forall i=1,2, \ldots, m$

where $d\left(\tilde{v}_{i j}, \tilde{v}_{j}^{ \pm}\right)=\left[1 / 3\left(\left(v_{i j 1}-v_{11}^{ \pm}\right)^{2}+\left(v_{i j 2}-v_{12}^{ \pm}\right)^{2}+\left(v_{i j 3}-v_{13}^{ \pm}\right)^{2}\right]^{0.5}\right.$.

Finally, the quality index of " $n$ " alternatives can be calculated as the reliability index $\left(R_{i}\right)$ and noise index $\left(N_{i}\right)$ (Eqs. 8 and 9 ). The higher reliability index, and lower noise index means that there is less noise within the measurement procedure.
Table 4 Descriptions of the experimental components associated with Fig. 3

\begin{tabular}{lll}
\hline Part number & Description & Technical information \\
\hline 1 & Load cell & $\begin{array}{c}\text { Crow-tail sensor with } \\
\text { HX711 amplifier }\end{array}$ \\
2 & Arduino micro-controller & (ATmega328) \\
3 & Analog to digital convertor & Digital I/O pins 24 \\
4 & Intermediate software & Lab view \\
\hline
\end{tabular}

$R_{i}=\frac{d_{i}^{+}}{d_{i}^{+}+d_{i}^{-}} \quad i=1,2, \ldots, m$

$N_{i}=\frac{d_{i}^{-}}{d_{i}^{+}+d_{i}^{-}} \quad i=1,2, \ldots, m$.

\subsection{Experimental setup}

The experimental components used in strain measurement are described in Table 4, including the technical information for each component.

In the experimental tests, a Crow-tail load cell sensor was employed. The HX711 amplifier was embedded in the kit, with high accuracy of a 24-bit analog-to-digital converter (ADC) as shown in Fig. 3a. The Crow-tail load cell has been designed for a weigh scale and industrial control applications with the accuracy of the mass sensor of $0-5 \mathrm{~kg}$ as shown in Fig. $3 \mathrm{~b}$. The calibration process for the selected sensor is implemented by applying the same mass $(40 \mathrm{~g})$ to guarantee that no noise is produced.

In the experimental tests, a $\mathrm{C}++$ program was provided and embedded in the Arduino controller. The measured data as outputs were collected from the controller by altering the inputs. In the next section, the details of strain measurements are explained.

\section{Result and discussion}

\subsection{Strain measurement}

The strain value was evaluated based on the mass, the cross-section area of the square aluminum bar and the module of elasticity employing the following equations:

The total stress was evaluated by Eq. 10:

$\sigma=\frac{F}{A}=\frac{m g}{A}$,

where $F(\mathrm{~N})$ stands for the applied force, $A\left(\mathrm{~m}^{2}\right)$ represents the cross-section area of the bar, and $m(\mathrm{~kg})$ is the mass. Equation 11 is used to calculate the strain value as follow: 
Fig. 3 a Crow-tail series sensor with HX711 amplifier, front and back view, b Square aluminum beam used in the tests with the attached load cell
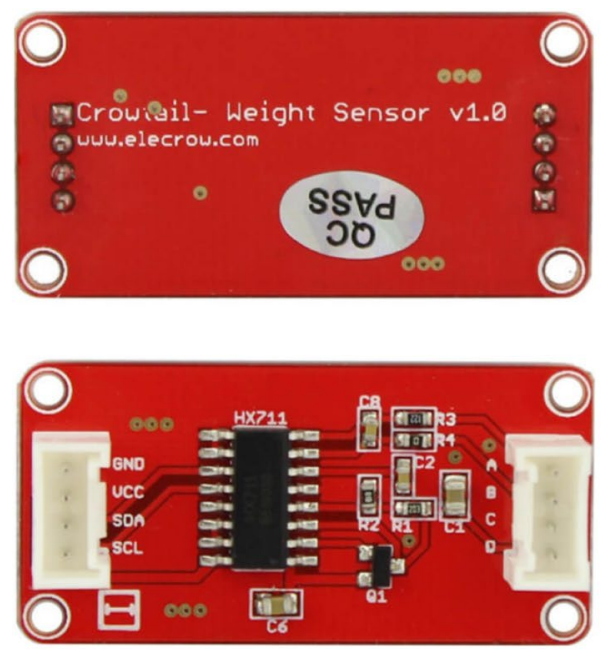

(a)

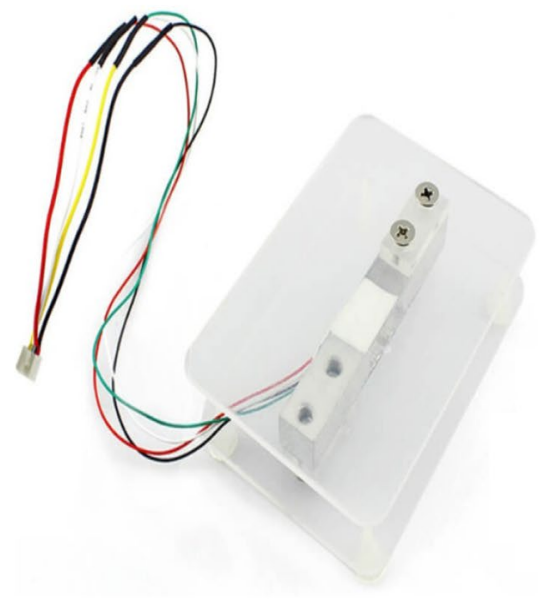

(b)

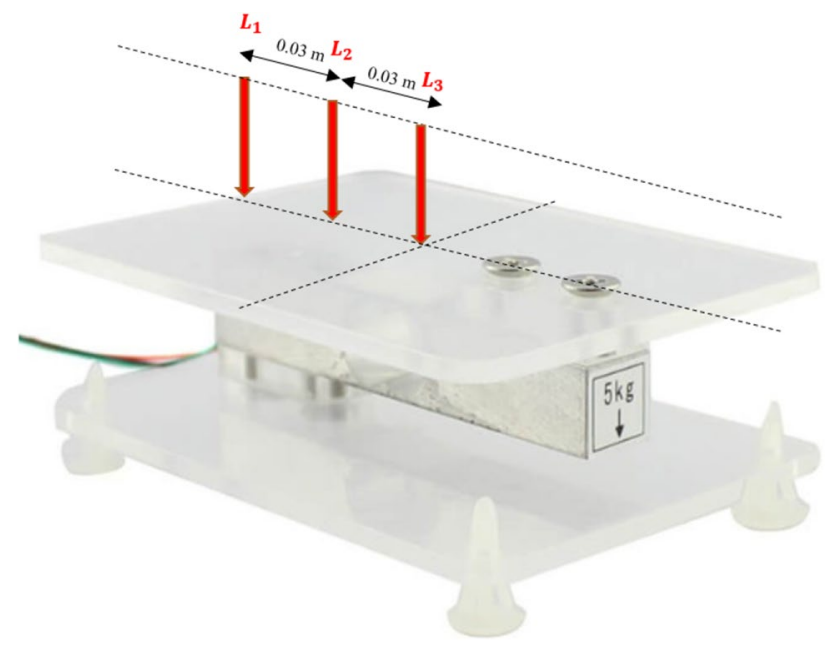

Fig. 4 Point of applied force on the strain gauge $\left(L_{1}, L_{2}\right.$, and $\left.L_{3}\right)$, including the gaps between the loads
$s=\frac{\sigma}{E}$

here $s$ and $\sigma$ are the strain (unitless) and the stress $\left(\mathrm{N} / \mathrm{m}^{2}\right)$, respectively. $E\left(\mathrm{~N} / \mathrm{m}^{2}\right)$ represents the module of elasticity of the aluminum. Three points of the applied forces with a gap of $0.03(\mathrm{~m})$ are considered as shown in Fig. 4. Both the $S / N$ ratio and the analysis of variance were applied to determine the significance and percentage of contribution of each parameter.

Table 5 summarizes the evaluation of outcomes, including the stresses and strains of all experimental tests. The tests were conducted for three measurements known as $m_{1}, m_{2}$, and $m_{3}$, for the same mass (40 g), which are obtained by Arduino micro-controller. This repetition enhances the accuracy of the strain measurements. The mass was evaluated according to the employed material, which is aluminum $\left(E_{A l}=69 \times 10^{9} \mathrm{MPa}\right)$.

Table 5 Strain measurement of the test cases based on the $L_{9}$ orthogonal array, using three types of masses

\begin{tabular}{|c|c|c|c|c|c|c|c|c|c|}
\hline Trial number & $m_{1}(\mathrm{~g})$ & $m_{2}(\mathrm{~g})$ & $m_{3}(\mathrm{~g})$ & Stress $1(\mathrm{~Pa})$ & Stress $2(\mathrm{~Pa})$ & Stress $3(\mathrm{~Pa})$ & Strain 1 & Strain 2 & Strain 3 \\
\hline 1 & 39.5 & 39.6 & 39.5 & 40.40 & 40.62 & 40.40 & $5.84 \mathrm{E}-08$ & $5.86 \mathrm{E}-08$ & $5.84 \mathrm{E}-08$ \\
\hline 2 & 38.9 & 38.8 & 38.8 & 39.09 & 39.87 & 39.87 & $5.76 \mathrm{E}-08$ & $5.74 \mathrm{E}-08$ & $5.74 \mathrm{E}-08$ \\
\hline 3 & 38.9 & 38.9 & 38.9 & 39.09 & 39.09 & 39.09 & $5.76 \mathrm{E}-08$ & $5.76 \mathrm{E}-08$ & $5.76 \mathrm{E}-08$ \\
\hline 4 & 40.9 & 40.6 & 41 & 41.46 & 41.81 & 41.68 & $6.05 \mathrm{E}-08$ & $6.01 \mathrm{E}-08$ & $6.07 \mathrm{E}-08$ \\
\hline 5 & 42.2 & 42 & 41.6 & 43.31 & 42.87 & 42.75 & $6.24 \mathrm{E}-08$ & $6.22 \mathrm{E}-08$ & $6.16 \mathrm{E}-08$ \\
\hline 6 & 42 & 41.7 & 41.5 & 42.80 & 42.22 & 42.78 & $6.22 \mathrm{E}-08$ & $6.17 \mathrm{E}-08$ & $6.14 \mathrm{E}-08$ \\
\hline 7 & 38 & 38.3 & 38.3 & 38.10 & 39.785 & 39.78 & $5.62 \mathrm{E}-08$ & $5.67 \mathrm{E}-08$ & 5.67E-08 \\
\hline 8 & 40 & 40 & 40.1 & 40.50 & 40.50 & 40.71 & $5.92 \mathrm{E}-08$ & $5.92 \mathrm{E}-08$ & $5.93 \mathrm{E}-08$ \\
\hline 9 & 39.5 & 38.8 & 41.7 & 40.40 & 39.87 & 42.21 & $5.84 \mathrm{E}-08$ & $5.74 \mathrm{E}-08$ & $6.17 \mathrm{E}-08$ \\
\hline
\end{tabular}


Table 6 Calculation of initial weights

\begin{tabular}{llll}
\hline & $S_{1}$ & $S_{2}$ & Initial weight \\
\hline Step weight & 0.5 & 0.5 & - \\
Accuracy & 1 & - & 0.5 \\
Precision & - & 1 & 0.5 \\
\hline
\end{tabular}

Table 7 Fuzzy rating of 9 trial numbers based on accuracy and precision

\begin{tabular}{lll}
\hline Trial number & Accuracy & Precision \\
\hline 1 & $\tilde{\mathrm{a}}_{2}(0,0.25,0.5)$ & $\tilde{\mathrm{a}}_{2}(0,0.25,0.5)$ \\
2 & $\tilde{\mathrm{a}}_{3}(0.25,0.5,0.75)$ & $\tilde{\mathrm{a}}_{2}(0,0.25,0.5)$ \\
3 & $\tilde{\mathrm{a}}_{3}(0.25,0.5,0.75)$ & $\tilde{\mathrm{a}}_{1}(0,0,0.25)$ \\
4 & $\tilde{\mathrm{a}}_{3}(0.25,0.5,0.75)$ & $\tilde{\mathrm{a}}_{3}(0.25,0.5,0.75)$ \\
5 & $\tilde{\mathrm{a}}_{5}(0.75,1,1)$ & $\tilde{\mathrm{a}}_{2}(0,0.25,0.5)$ \\
6 & $\tilde{\mathrm{a}}_{5}(0.75,1,1)$ & $\tilde{\mathrm{a}}_{4}(0.5,0.75,1)$ \\
7 & $\tilde{\mathrm{a}}_{5}(0.75,1,1)$ & $\tilde{\mathrm{a}}_{4}(0.5,0.75,1)$ \\
8 & $\tilde{\mathrm{a}}_{1}(0,0,0.25)$ & $\tilde{\mathrm{a}}_{1}(0,0,0.25)$ \\
9 & $\tilde{\mathrm{a}}_{1}(0,0,0.25)$ & $\tilde{\mathrm{a}}_{5}(0.75,1,1)$ \\
\hline
\end{tabular}

\subsection{Optimization process}

The initial weight of each criterion, based on their classifications (Fig. 2), is calculated using AHP, and the results are tabulated in Table 6.

Fuzzy rating for accuracy and precision of nine experiments is implemented. Based on their seriousness, different level of fuzzy rating is considered as triangular fuzzy numbers, and the results are tabulated in Table 7 .

Based on the literature, the regulative factors are selected as $\alpha=\beta=1.25, \lambda_{1}=\lambda_{2}=1.5$ [7]. The varied weight of each criterion was determined in Table 7 via Eqs. 1 and 2. The reliability index was also evaluated for all trial numbers (Eq. 8) as shown in Table 8. Then, fuzzy evaluation was used to determine the results of nine experiments for accuracy and precision as shown in Table 9 using Eq. 3. It is found
Table 9 Fuzzy evaluating results of nine experiments for accuracy and precision

\begin{tabular}{lll}
\hline Trial number & Accuracy & Precision \\
\hline 1 & $(0,0.125,0.25)$ & $(0,0.125,0.25)$ \\
2 & $(0.11,0.22,0.33)$ & $(0,0.14,0.28)$ \\
3 & $(0.0875,0.175,0.2625)$ & $(0,0,0.162)$ \\
4 & $(0.125,0.25,0.375)$ & $(0125,0.25,0.375)$ \\
5 & $(0.45,0.6,0.6)$ & $(0,0.1,0.2)$ \\
6 & $(0.45,0.6,0.6)$ & $(0.2,0.3,0.4)$ \\
7 & $(0.45,0.6,0.6)$ & $(0.2,0.3,0.4)$ \\
8 & $(0,0,0.0875)$ & $(0,0,0.162)$ \\
9 & $(0,0,0.125)$ & $(0.375,0.5,0.5)$ \\
\hline
\end{tabular}

Table 10 Reliability and Noise indices of nine experiments based on accuracy and precision

\begin{tabular}{llllll}
\hline Trial number & $d_{i}^{+}$ & $d_{i}^{-}$ & $d_{i}^{+}+d_{i}^{-}$ & $R_{i}$ & $N_{i}$ \\
\hline 1 & 0.8744 & 0.3227 & 1.1972 & 0.7304 & 0.2695 \\
2 & 0.7681 & 0.4183 & 1.1865 & 0.6474 & 0.3525 \\
3 & 0.8833 & 0.2828 & 1.1661 & 0.7574 & 0.2425 \\
4 & 0.6346 & 0.5400 & 1.1746 & 0.5402 & 0.4597 \\
5 & 0.4948 & 0.6836 & 1.1784 & 0.4199 & 0.5800 \\
6 & 0.3026 & 0.8654 & 1.1680 & 0.2590 & 0.7409 \\
7 & 0.3026 & 0.8654 & 1.1680 & 0.2590 & 0.7409 \\
8 & 1.0246 & 0.1443 & 1.1690 & 0.8765 & 0.1234 \\
9 & 0.6336 & 0.5342 & 1.1678 & 0.5425 & 0.4574 \\
\hline
\end{tabular}

that trial number 8 has the highest reliability index based on accuracy and precision as shown in Table 10 after all calculations (Fig. 5).

A sample of calculation is performed here for trial test 1 , for evaluation of accuracy employing Eq. 3 . The result can be evaluated as follow.

Sample of calculation:

$(0,0.25,0.5) \times 0.5=(0,0.125,0.25)$
Table 8 Calculation of varied weight based on variable weight profit factor

\begin{tabular}{|c|c|c|c|c|c|c|}
\hline \multirow[t]{2}{*}{ Trial number } & \multicolumn{3}{|c|}{ Accuracy } & \multicolumn{3}{|c|}{ Precision } \\
\hline & $x_{j}$ & $s_{j}\left(x_{j}\right)$ & $w_{j}\left(x_{j}\right)$ & $x_{j}$ & $s_{j}\left(x_{j}\right)$ & $w_{j}\left(x_{j}\right)$ \\
\hline 1 & $\tilde{a}_{2}$ & 1.25 & 0.50 & $\tilde{a}_{2}$ & 1.25 & 0.50 \\
\hline 2 & $\tilde{a}_{3}$ & 1.00 & 0.44 & $\tilde{a}_{2}$ & 1.25 & 0.56 \\
\hline 3 & $\tilde{\mathrm{a}}_{3}$ & 1.00 & 0.35 & $\tilde{\mathbf{a}}_{1}$ & 1.87 & 0.65 \\
\hline 4 & $\tilde{a}_{3}$ & 1.00 & 0.50 & $\tilde{\mathrm{a}}_{3}$ & 1.00 & 0.50 \\
\hline 5 & $\tilde{a}_{5}$ & 1.87 & 0.60 & $\tilde{a}_{2}$ & 1.25 & 0.40 \\
\hline 6 & $\tilde{\mathrm{a}}_{5}$ & 1.87 & 0.60 & $\tilde{\mathbf{a}}_{4}$ & 1.25 & 0.40 \\
\hline 7 & $\tilde{\mathrm{a}}_{5}$ & 1.87 & 0.60 & $\tilde{\mathbf{a}}_{4}$ & 1.25 & 0.40 \\
\hline 8 & $\tilde{a}_{1}$ & 1.00 & 0.35 & $\tilde{\mathbf{a}}_{1}$ & 1.87 & 0.65 \\
\hline 9 & $\tilde{a}_{1}$ & 1.87 & 0.50 & $\tilde{\mathbf{a}}_{5}$ & 1.87 & 0.50 \\
\hline
\end{tabular}




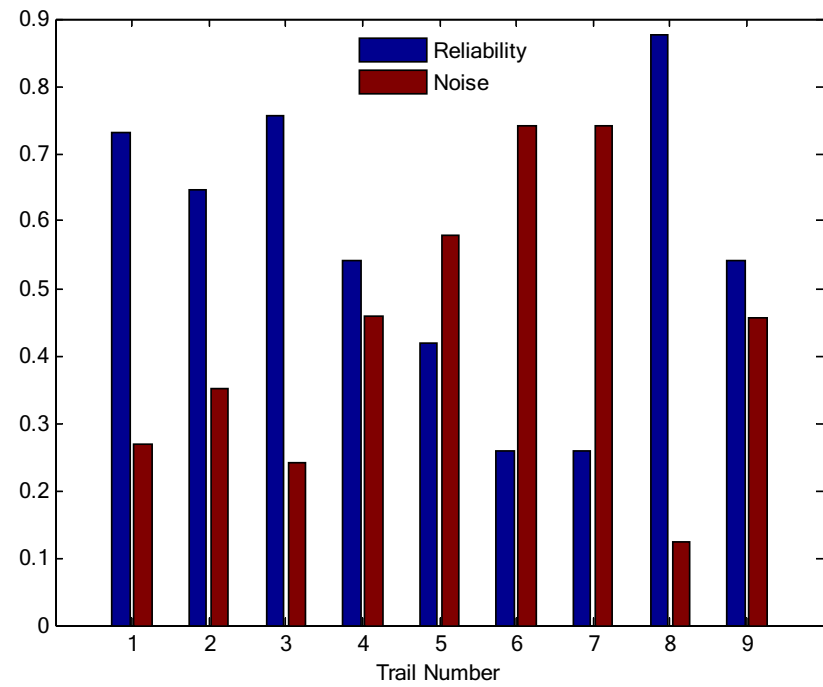

Fig. 5 Comparison of Reliability and Noise indices at 9 levels applying the data of Table 10

Reliability index $\left(R_{i}\right)$ and Noise index $\left(N_{i}\right)$ are calculated for nine experimental tests and shown in Table 10. The maximum reliability index is 0.8765 and the minimum noise index is 0.1234 which are related to experiment number 8 . Although, the best set of parameters are $P_{1}$ at level $3, P_{2}$ at level 2 , and $P_{3}$ at level $1 \mathrm{lt}$, the calculation of Signal to Noise ratio will determine the optimum level of individual parameters with higher reliability index and lower noise index. The distance of each alternative or experiment based on the orthogonal array can also be calculated using Eq. 8:

$$
\begin{aligned}
d_{\text {Accuracy }}^{+} & =\left[1 / 3\left((0-0.6)^{2}+(0.125-0.6)^{2}+(0.25-0.6)^{2}\right)\right]^{0.5} \\
& =0.4858 \\
d_{\text {precision }}^{+} & =\left[1 / 3\left((0.5-0)^{2}+(0.5-0.125)^{2}+(0.5-0.25)^{2}\right)\right]^{0.5} \\
& =0.3886
\end{aligned}
$$

So the distance of trial 1 is calculated as:

$d_{1}^{+}=0.4858+0.3886 \cong 0.8744$

After determining the Reliability and Noise Indexes, the next step is to apply $S / N$ ratios to find the significant parameters and their optimum level in order to find the minimum noise (high accuracy and precision). To reduce the noise, "The smaller the better" is chosen for the Noise index. The calculated results for temperature measurement and $S / N$ ratio have been determined in Table 11 . The value for the $S / N$ was calculated based on Eqs. 12 and 13 for each experiment. MSD stands for mean squared deviation.

$M S D=\frac{1}{N} \sum_{i=1}^{n} y_{i}^{2}$
Table $11 S / N$ ratio for all test cases

\begin{tabular}{lllc}
\hline Trial number & $N_{l}$ & $M S D$ & $S / N$ ratio \\
\hline 1 & 0.2695 & 0.06 & 11.70 \\
2 & 0.3525 & 0.09 & 10.45 \\
3 & 0.2425 & 0.04 & 13.97 \\
4 & 0.4597 & 0.16 & 7.95 \\
5 & 0.5800 & 0.25 & 6.02 \\
6 & 0.7409 & 0.49 & 3.09 \\
7 & 0.7409 & 0.49 & 3.09 \\
8 & 0.1234 & 0.01 & 20 \\
9 & 0.4574 & 0.16 & 7.95 \\
\hline
\end{tabular}

Table 12 The response table of $S / N$ ratio

\begin{tabular}{lccc}
\hline & $P_{1}$ :Temperature & $P_{2}$ : length of wire & $\begin{array}{l}P_{3} \text { : point of } \\
\text { application }\end{array}$ \\
\hline Level 1 & 12.04 & 7.58 & 11.59 \\
Level 2 & 5.69 & 12.15 & 8.79 \\
Level 3 & 10.35 & 8.34 & 7.69 \\
Difference $|\Delta \mathrm{T}|$ & 6.35 & 4.57 & 3.90 \\
\hline
\end{tabular}

$S / N=-10 \log (M S D)$

From the collecting data in Table 11, the average $S / N$ ratio for the response table can be calculated. The responses are summarized in Table 12 to determine the optimal levels of the selected parameters.

The difference between the levels is defined as $|\boldsymbol{\Delta} \mathbf{T}|$. The larger value of $|\Delta T|$ demonstrates the significance of the parameter. It is clear that $P_{1}$ is the most significant parameter followed by $P_{3}$, and $P_{2}$, respectively. The optimum set of parameters can be evaluated from Table 12 by selecting the highest level of $S / N$ for each parameter. The best results are $P_{1}$ at level $1, P_{2}$ at level 2 , and $P_{3}$ at level 1 . This is clearly highlighted in a bar chart as shown in Fig. 6 . The results reveal that by setting the temperature at $23^{\circ} \mathrm{C}$, length of wires at $10 \mathrm{~cm}$, and point of application at $1 \mathrm{~cm}$, the highest reliability is achievable with minimum noise. After setting the controllable parameters to their optimal levels, the final result for the selected mass was $40 \mathrm{~g}$.

Once the optimum level of individual parameters was determined, the degrees of freedom, the sum of squares, the variance, $F$ ratio, the pure sum of a square and their contribution are calculated via ANOVA. The individual sum of a square and the total sum of the square are calculated based on Eqs. 14-16: 


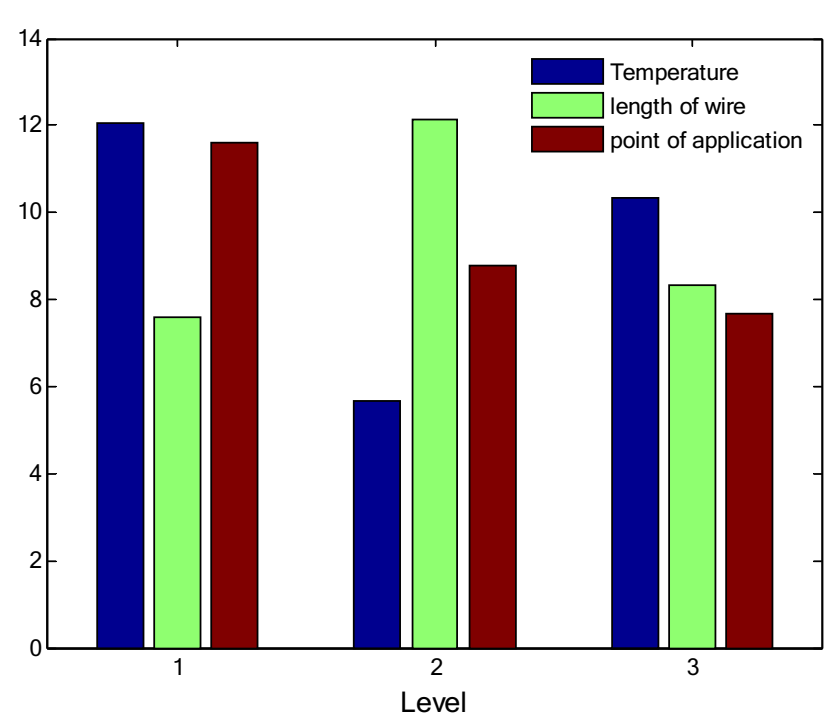

Fig. 6 Comparison of Temperature, the length of wire and the point of application at three levels based on Table 12

$$
\begin{aligned}
& S S_{P_{i}}=\sum_{i=1}^{K_{A}}\left(\frac{A_{i}^{2}}{n_{A_{i}}}\right)-\frac{\left(\sum_{i=1}^{N} x_{i}\right)^{2}}{N}, \\
& S S_{T}=\sum_{i=1}^{K_{A}} x_{i}^{2}-\frac{\left(\sum_{i=1}^{N} x_{i}\right)^{2}}{N} \\
& S S_{E}=\left(S S_{T}-\sum_{i=1}^{K_{A}} S S_{P_{i}}\right)
\end{aligned}
$$

where $A_{i}$ is the average temperature for each level, $n_{A_{i}}$ stands for the number of levels, $x_{i}$ represents the temperature value in each experiment, $N$ is the number of experiments and $k_{A}$ represents the number of parameters. For the calculation of the degree of freedom of each level $\left(F_{i}\right)$ and the total degree of freedom $\left(F_{t}\right)$ the following equations were used, respectively:

$F_{i}=x_{i}-1$,

$F_{t}=N-1$.

Thus, to determine the error of the degree of freedom, Eq. 19 is applied as follow:

$$
F_{e}=F_{t}-\sum_{i=1}^{K_{A}} F_{i}
$$

The variance of the individual parameter $\left(V_{P_{i}}\right)$ and the variance of error $\left(V_{e}\right)$ are calculated as follow:

$V_{P_{i}}=\frac{S S_{P_{i}}}{F_{i}}$

$V_{e}=\frac{S S_{e}}{F_{e}}$

Finally, the percentage of contribution of each parameter $\left(P_{P_{i}}\right)$ can be determined by Eq. 22 .

$P_{P_{i}}=\frac{S S_{P_{i}}}{S S_{T}}$

$S_{e}=S_{T}-S_{P_{1}}-S_{P_{2}}-S_{P_{3}}$

By using Eqs. 10-18, the weight or percentage of contribution of each parameter is calculated, as shown in Table 13 which is associated with Fig. 7. The results indicate that the temperature with $67.36 \%$ has the highest contribution, followed by the length of wire with $17.15 \%$ and the point of application with $15.48 \%$. According to Eqs. 18 and 19, the amount of error for degree of freedom is 2. Also, based on Eq. 23 the error for both sum of square and the percentage of contribution is zero. The

Table 13 Analysis of variance (ANOVA) for the selected parameters

\begin{tabular}{lllc}
\hline & $F$ & $S S$ & $P(\%)$ \\
\hline$P_{1}:$ Temperature & 2 & 0.1398 & 67.36 \\
$P_{2}:$ length of wire & 2 & 0.03561 & 17.15 \\
$P_{3}:$ point of application & 2 & 0.03214 & 15.48 \\
Error & 2 & 0 & 0 \\
Total & 8 & 0.2076 & 100 \\
\hline
\end{tabular}

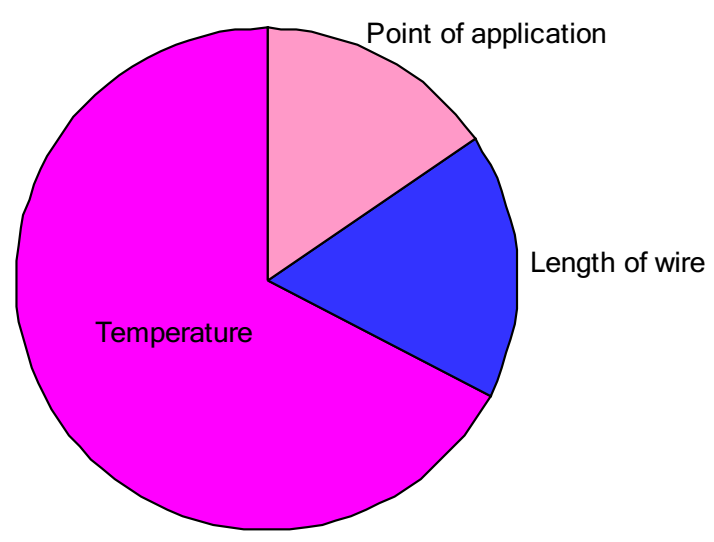

Fig. 7 Pie chart of contribution of Variance (ANOVA) for the three selected parameters, including Temperature, the length of wire and the point of application 
combination of Taguchi and TOPSIS was implemented. The determination of an experiment with the highest reliability of strain gauge was considered and experiment number 8 is nominated with the highest reliability index. In addition, the contribution of Taguchi led to the determination of significant parameters and their percentage of contribution affecting the reliability of strain gauges for different applications.

\section{Conclusion}

Combination of Taguchi and FAHP with TOPSIS offers a well-organized use approach for multi-objective problems. Determining the highest reliability index in strain measurement is the objective of this study. By using the Taguchi method, the significant controllable factors and their optimum levels were identified. Two common concepts in measurement were selected as a different criterion to evaluate the reliability and noise index. The proposed method was applied for three different controllable parameters, namely the temperature, the length of wire, and the point of application. For rating the seriousness of each criterion, a fuzzy evaluation is considered by defining five different levels. TOPSIS was chosen to rank the order of nine experiments. According to the TOPSIS approach, it is found that the highest reliability index is related to trial number 8 . The best set of parameters are $P_{1}$ at level $3, P_{2}$ at level 2 , and $P_{3}$ at level 1 with $88 \%$ reliability index and $12 \%$ noise index. The optimum levels are $P_{1}$ at level $1, P_{2}$ at level 2 , and $P_{3}$ at level 1 and the temperature is the most significant parameters with $67.36 \%$ contribution, which alters the accuracy and precision in strain measurement.

\section{Compliance withy ethical standards}

Conflict of interest The authors declare that they have no conflict of interest.

\section{References}

1. Alam MM, Zheng Q, Derakhshandeh JF, Rehman S, Ji C, Zafar $F$ (2018) On forces and phase lags between vortex sheddings from three tandem cylinders. Int J Heat Fluid Flow 60:117-135

2. Amer Y, Moayyedian M, Hajiabolhasani Z, Moayyedian L (2012) Reducing warpage in injection moulding processes using Taguchi method approach: ANOVA. Doctoral dissertation, ACTA Press, Calgary

3. Amer Y, Moayyedian M, Hajiabolhasani Z, Moayyedian L (2013) Improving injection moulding processes using experimental design. Doctoral dissertation, WASET-World Academy of Science Engineering

4. Arasaratnam P, Sivakumaran KS, Tait MJ (2011) True Stress-True Strain models for structural steel elements. Int Sch Res Netw ISRN Civ Eng 2011:11. https://doi.org/10.5402/2011/656401

5. Ashutosh Kumar A, Chaturvedi SK, Chaturvedi V, Yadaw RC (2012) Design studies and optimization of position of strain gauge. Int J Sci Eng Res 3(10):1-4

6. Chen MY, Tzeng HW, Chen YC, Chen SC (2008) The application of fuzzy theory for the control of weld line positions in injection-molded part. ISA Trans 47:119-126

7. Cheng J, Feng Tan, Wei JW (2008) Optimization of injection mold based on fuzzy moldability evaluation. J Mater Process Technol 208:222-228

8. Davis JR (2004) Tensile testing, 2nd edn. ASM International, Materials Park

9. Derakhshandeh JF, Arjomandi M, Dally B, Cazzolato B (2014) The effect of arrangements of two circular cylinders on the maximum efficiency of vortex- induced vibration power using a scale-adaptive simulation model. J Fluids Struct 49:654-666

10. Derakhshandeh JF, Arjomandi M, Dally B, Cazzolato B (2014) Effect of a rigid wall on the vortex induced vibration of two staggered cylinders. J Renew Sustain Energy Rev 6:033114

11. Derakhshandeh JF, Arjomandi M, Cazzolato B, Dally B (2014) Experimental and computational investigation of wake induced vibration. In: 19th Australasian fluid mechanics conference (AFMC), Melbourne, Australia

12. Derakhshandeh JF, Arjomandi M, Dally B, Cazzolato B (2015) Harnessing hydro-kinetic energy from wake induced vibration using virtual mass spring damper system. J Ocean Eng 108:115-128

13. Derakhshandeh JF, Arjomandi M, Dally B, Cazzolato B (2016) Flow-induced vibration of an elastically mounted airfoil under the influence of oncoming vortices. J Exp Therm Fluid Sci 74:58-72

14. Figliola RS, Beasley D (2015) Theory and design for mechanical measurements. Wiley, New York

15. Geethapriyan T, Muthuramalingam T, Kalaichelvan K (2019) Influence of process parameters on machinability of Inconel 718 by electrochemical micromachining process using TOPSIS technique. Arab J Sci Eng 44(9):7945-7955

16. Gu L (2001) A comparison of polynomial based regression models in vehicle safety analysis, in Diaz. In: ASME design engineering technical conferences-design automation conference, ASME, Pittsburgh, PA, USA, September 9-12, DAC-21063

17. He W, Zhang YF, Lee KS, Fuh JYH, Nee AYC (1998) Automated process parameter resetting for injection moulding: a fuzzy-neuro approach. J Intell Manuf 9:17-27

18. Huo J, Liu S, Wang Y, Muthuramalingam T, Pi VN (2019) Influence of process factors on surface measures on electrical discharge machined stainless steel using TOPSIS. Mater Res Exp 6(8):086507

19. Li X, Zhang R, Yu W, Wang K, Wei J, Wu D, Cao A, Li Z, Cheng Y, Zheng Q, Ruoff RS (2012) Stretchable and highly sensitive graphene-on-polymer strain sensors. Sci Rep 2:870

20. Lin MW, Rogers CA (1994) Bonding layer effects on the actuation mechanism of an induced strain actuator/substructure system. SPIE 2190:658-670

21. Lindsey LH, Kirstin P, Metin S (2017) Soft actuators for small-scale robotics. Adv Mater 29(13):1603483

22. Lord DJ, Morrel RM (2010) Elastic modules measurement-obtaining reliable data from the tensile test. Metrologia 47:502-515

23. Lu N, Lu C, Yang S, Rogers J (2012) Highly sensitive skin-mountable strain gauges based entirely on elastomers. Adv Funct Mater 22:4044-4050 
24. Meral G, Sarıkaya M, Dilipak H, Şeker U (2015) Multi-response optimization of cutting parameters for hole quality in drilling of AISI 1050 steel. Arab J Sci Eng 40(12):3709-3722

25. Moayyedian M, Abhary K, Marian R (2015) Improved gate system for scrap reduction in injection molding processes. Proc Manuf $2: 246-250$

26. Moayyedian M, Abhary K, Marian R (2016) The analysis of defects prediction in injection molding. World Acad Sci Eng Technol Int J Mech Aerosp Ind Mechatron Manuf Eng 10(12):1876-1879

27. Moayyedian M, Abhary K, Marian R (2016) Gate design and filling process analysis of the cavity in injection molding process. Adv Manuf 4(2):123-133

28. Moayyedian M, Abhary K, Marian R (2017) The analysis of short shot possibility in injection molding process. Int J Adv Manuf Technol 191(9-12):3977-3989

29. Moayyedian M, Derakhshandeh JF, Sherif S (2018) Experimental investigations of significant parameters of strain measurement employing Taguchi method. SN Appl Sci 1-9

30. Moayyedian M, Abhary K, Marian R (2018) Optimization of injection molding process based on fuzzy quality evaluation and Taguchi experimental design. CIRP J Manuf Sci Technol 21:150-160

31. Moayyedian M (2018) Intelligent optimization of mold design and process parameters in injection molding. Springer, New York

32. Moayyedian M, Mamedov A (2019) Multi-objective optimization of injection molding process for determination of feasible moldability index. Proc CIRP 84:769-773
33. Motra HB, Hildebrandb J, Dimmig-Osburg A (2014) Assessment of strain measurement techniques to characterize mechanical properties of structural steel. Eng Sci Technol Int J 17:260-269

34. Ponnusamy P, Masood SH, Ruan D, Palanisamy S, Rashid R (2018) High strain rate dynamic behaviour of AISi12 alloy processed by selective laser melting. Int J Adv Manuf Technol 97(1-4):1023-1035

35. Sarıkaya M, Güllü A (2015) Multi-response optimization of minimum quantity lubrication parameters using Taguchi-based grey relational analysis in turning of difficult-to-cut alloy Haynes 25. J Clean Prod 91:347-357

36. Sarıkaya M, Yılmaz V, Güllü A (2016) Analysis of cutting parameters and cooling/lubrication methods for sustainable machining in turning of Haynes 25 superalloy. J Clean Prod 133:172-181

37. Singh J, Kumar A, Sharma ND, Bandyopadhyay AK (2011) Reliability and long term stability of a digital pressure gauge (DPG) used as a standard-a case study. Mapan 26(2):115-124

38. Sirohi J, Chopra I (2000) Fundamental understanding of piezoelectric strain sensors. J Intell Mater Syst Struct 11:246-257

39. Wang GG, Shan S (2007) Review of metamodeling techniques in support of engineering design optimization. Trans ASME 129:370-380

40. Willink R (2007) On the uncertainty of the mean of digitized measurement. Metrologia 44:73-81

Publisher's Note Springer Nature remains neutral with regard to jurisdictional claims in published maps and institutional affiliations. 